Supporting Information

\title{
Gear Alignments due to Hydrogen-Bonded Networks in a Crystal Structure of Resorcyltriptycene Hydrate and its Transformation to a non-Gearing Anhydrate Crystal by Heating
}

\author{
Noriyuki Tanaka, ${ }^{a}$ Yusuke Inagaki, ${ }^{a}$ Kentaro Yamaguchi, ${ }^{b}$ and Wataru Setaka ${ }^{*, a}$ \\ aDivision of Applied Chemistry, Faculty of Urban Environmental Sciences, Tokyo Metropolitan \\ University, 1-1 Minami-Osawa, Hachioji, Tokyo 192-0397, Japan \\ ${ }^{b}$ Faculty of Pharmaceutical Sciences at Kagawa Campus, Tokushima Bunri University, 1314-1 \\ Shido, Sanuki, Kagawa 769-2193, Japan \\ E-mail:wsetaka@tmu.ac.jp
}

(Total 14 pages including this cover page)

$\begin{array}{llll}\text { 1. Instruments and Software } & \ldots & \mathrm{S} 2\end{array}$

$\begin{array}{llll}\text { 2. Copies of NMR Spectra for All New Compounds } & \ldots & \text { S3 }\end{array}$

$\begin{array}{llll}\text { 3. Details of X-ray Crystallography } & \ldots & \text { S9 }\end{array}$

$\begin{array}{lllll}\text { 4. Temperature dependent } & { }^{1} \mathrm{H} & \text { NMR Study of } 1 & \ldots & \text { S12 }\end{array}$

$\begin{array}{llll}\text { 5. Solid-state }{ }^{13} \text { C CP/MAS NMR Study } & \ldots & \text { S13 }\end{array}$

$\begin{array}{llll}\text { 6. Temperature dependent PXRD Study } & \ldots & \text { S14 }\end{array}$ 


\section{Instruments and Software}

Single Crystal X-ray Crysrallography was carried out using RIGAKU XtaLAB mini for Crystals I and II. Diffraction data were indexed by RIGAKU CrystalClear software. For Crystal III, X-ray Crysrallography was carried out using Bruker APEX II diffractometer. All of the structural refinements were carried out using Shelxl software with WinGX Software. Molecular and Crystal structure was drawn using CCDC Mercury software.

Powder X-ray Diffraction (PXRD) was measured using RIGAKU SmartLab powder diffract meter equipped with a temperature controller. The observed diffractions were treated with RIGAKU SmartLab Studio II software. Simulation of the powder diffraction pattern from the single-crystal diffraction data was carried out using CCDC Mercury Software.

Solution NMR spectra was recorded using Bruker Avance 500 spectrometer equipped with a temperature controller.

Solid-state NMR spectra was recorded using JEOL ECA400 spectrometer.

DSC and TG analysis were carried out using HITATCH TA7000 analyzer.

HRMS was measured by Bruker microTOF spectrometer. 


\section{Copies of Spectra for All New Compounds}

\section{a. 9-(3,5-dihydroxyphenyl)triptycene (1)}

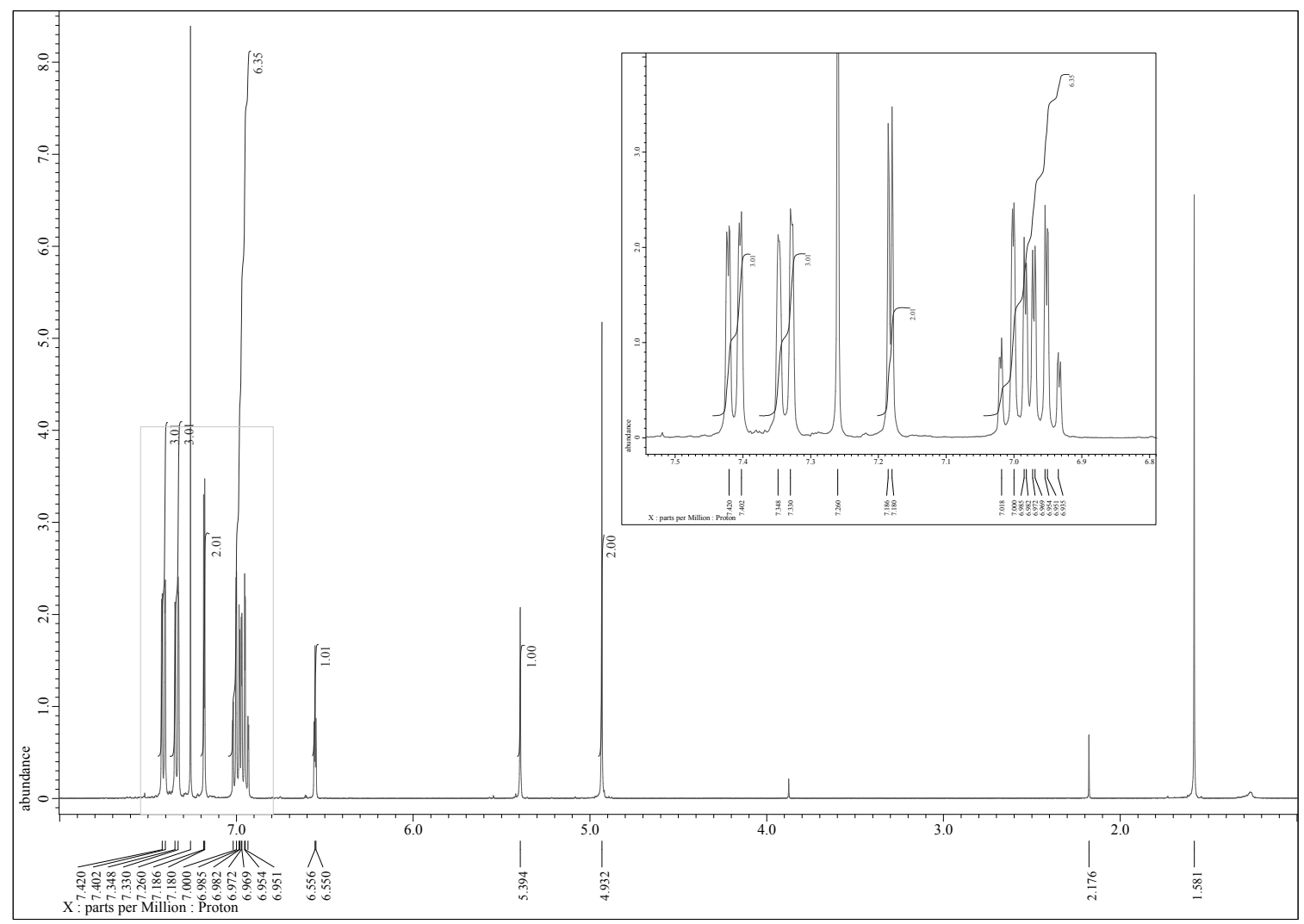

Fig. S1. ${ }^{1} \mathrm{H}$ NMR spectrum of 9-(3,5-dihydroxyphenyl)triptycene (1) in $\mathrm{CDCl}_{3}$.

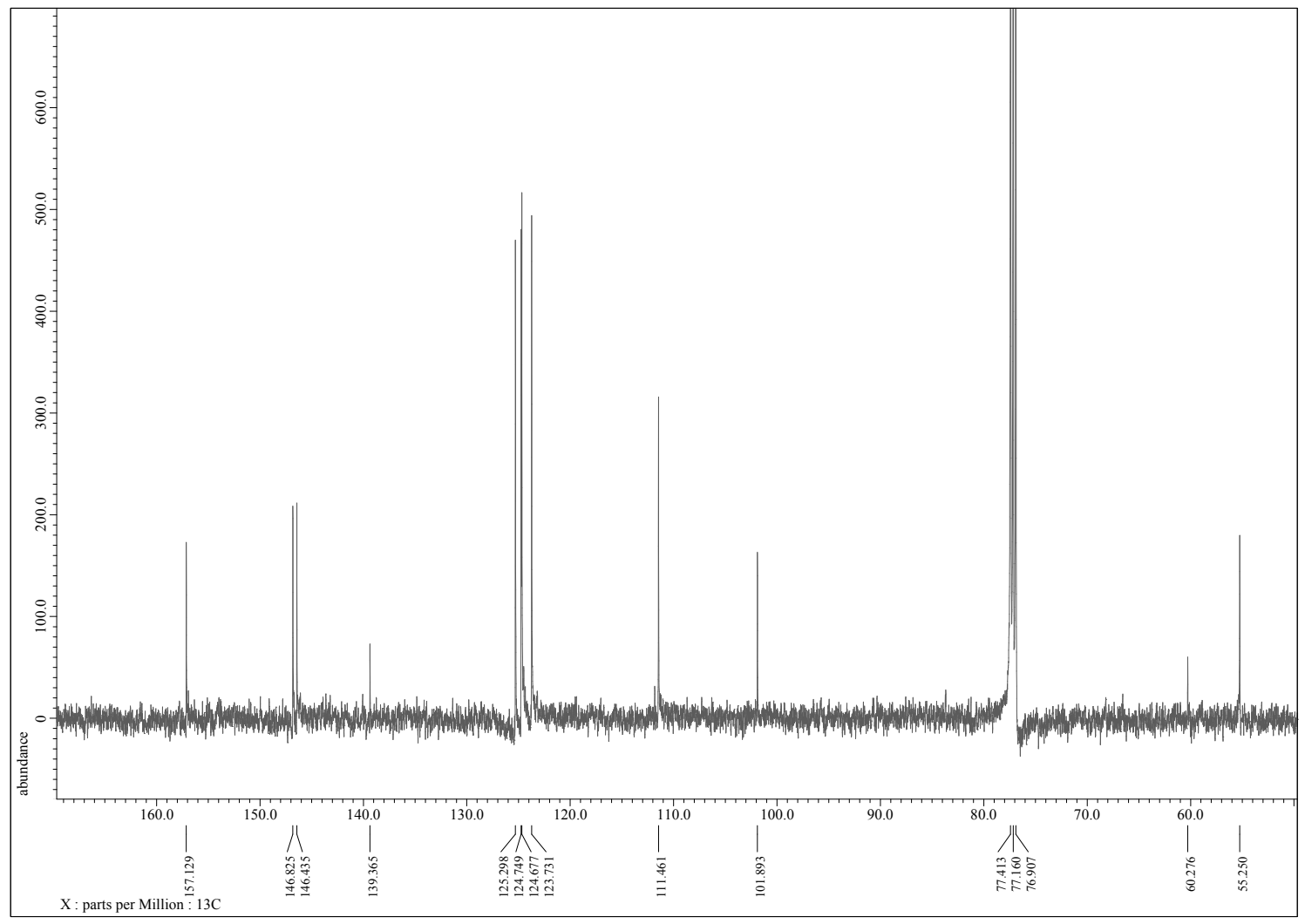

Fig. S2. ${ }^{13} \mathrm{C}$ NMR spectrum of 9-(3,5-dihydroxyphenyl)triptycene (1) in $\mathrm{CDCl}_{3}$. 

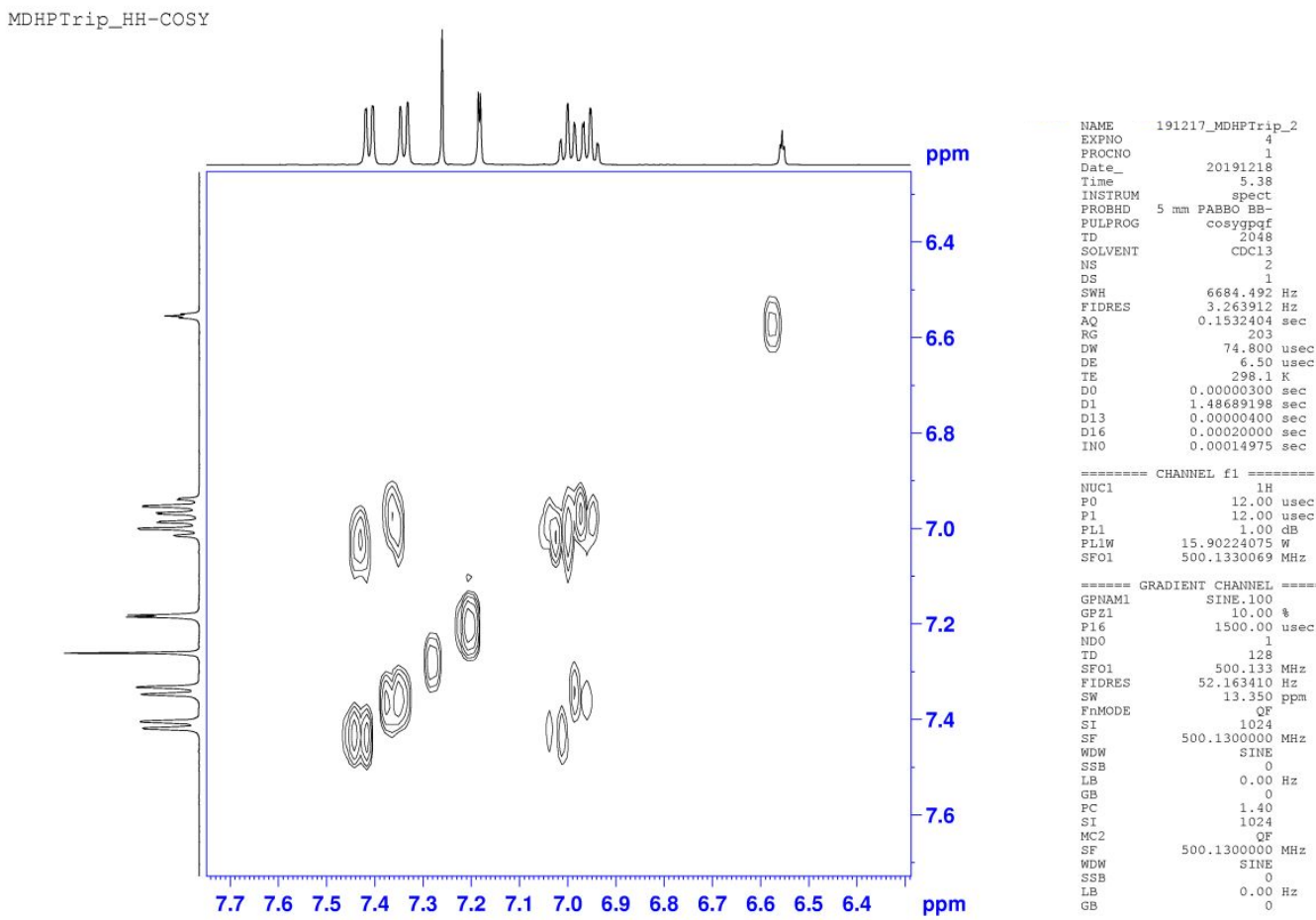

Fig. S3. ${ }^{1} \mathrm{H}-{ }^{1} \mathrm{H}$ COSY NMR spectrum (aromatic region) of 9-(3,5-dihydroxyphenyl)triptycene (1).
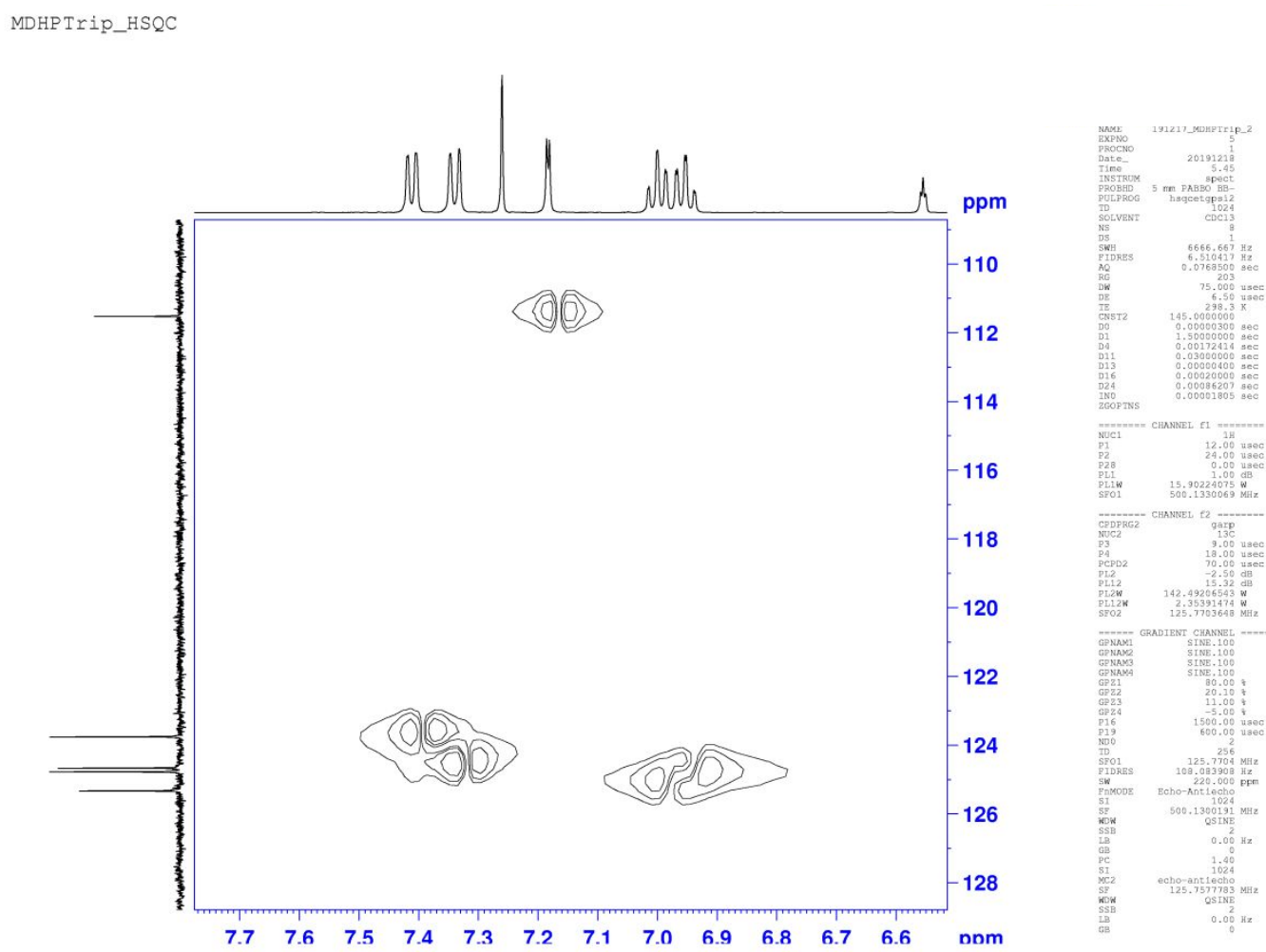

Fig. S4. ${ }^{1} \mathrm{H}-{ }^{13} \mathrm{C}$ HSQC NMR spectrum (aromatic region) of 9-(3,5-dihydroxyphenyl)triptycene (1). 

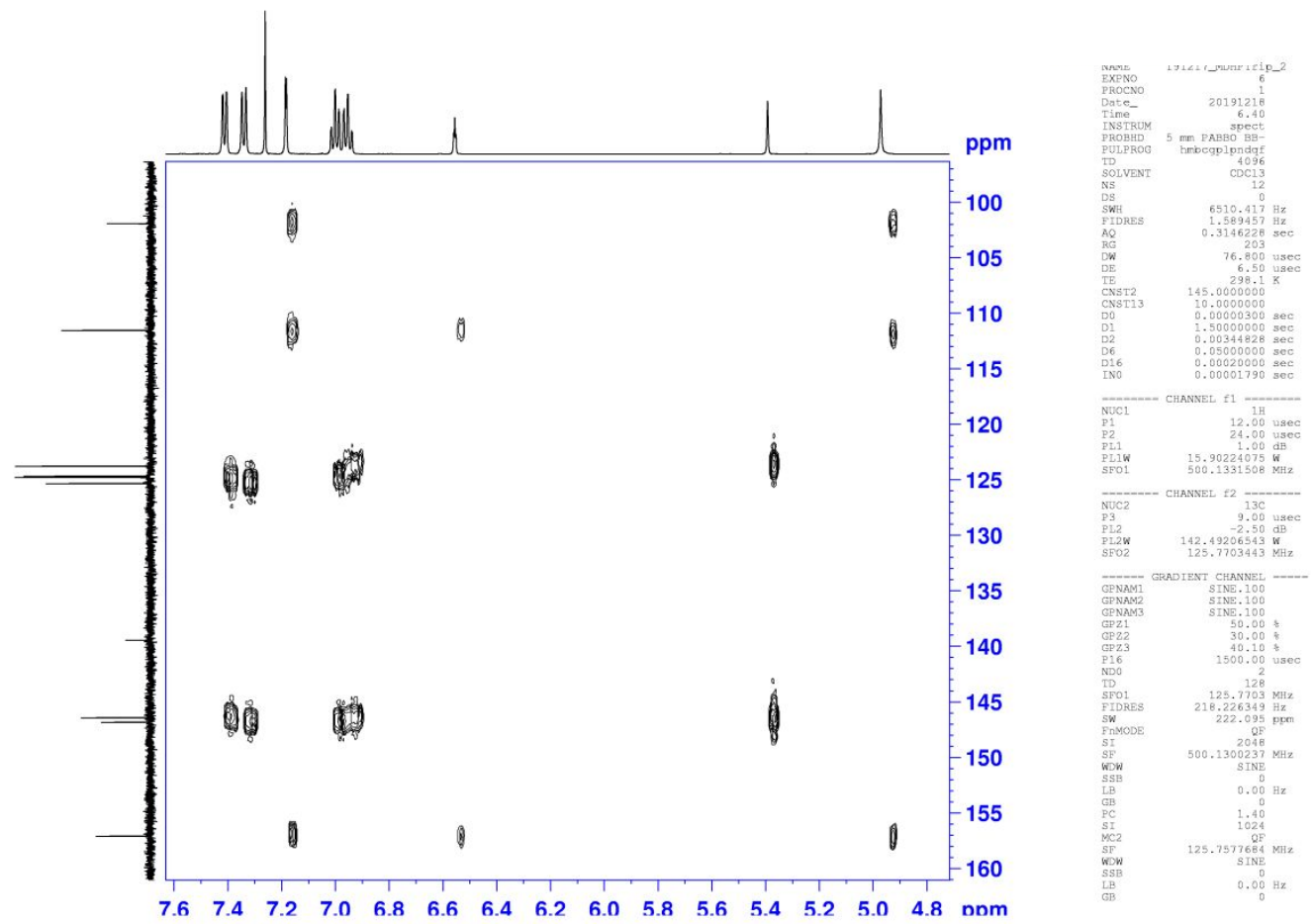

Fig. S5. ${ }^{1} \mathrm{H}-{ }^{13} \mathrm{C}$ HMBC NMR spectrum of 9-(3,5-dihydroxyphenyl)triptycene (1).

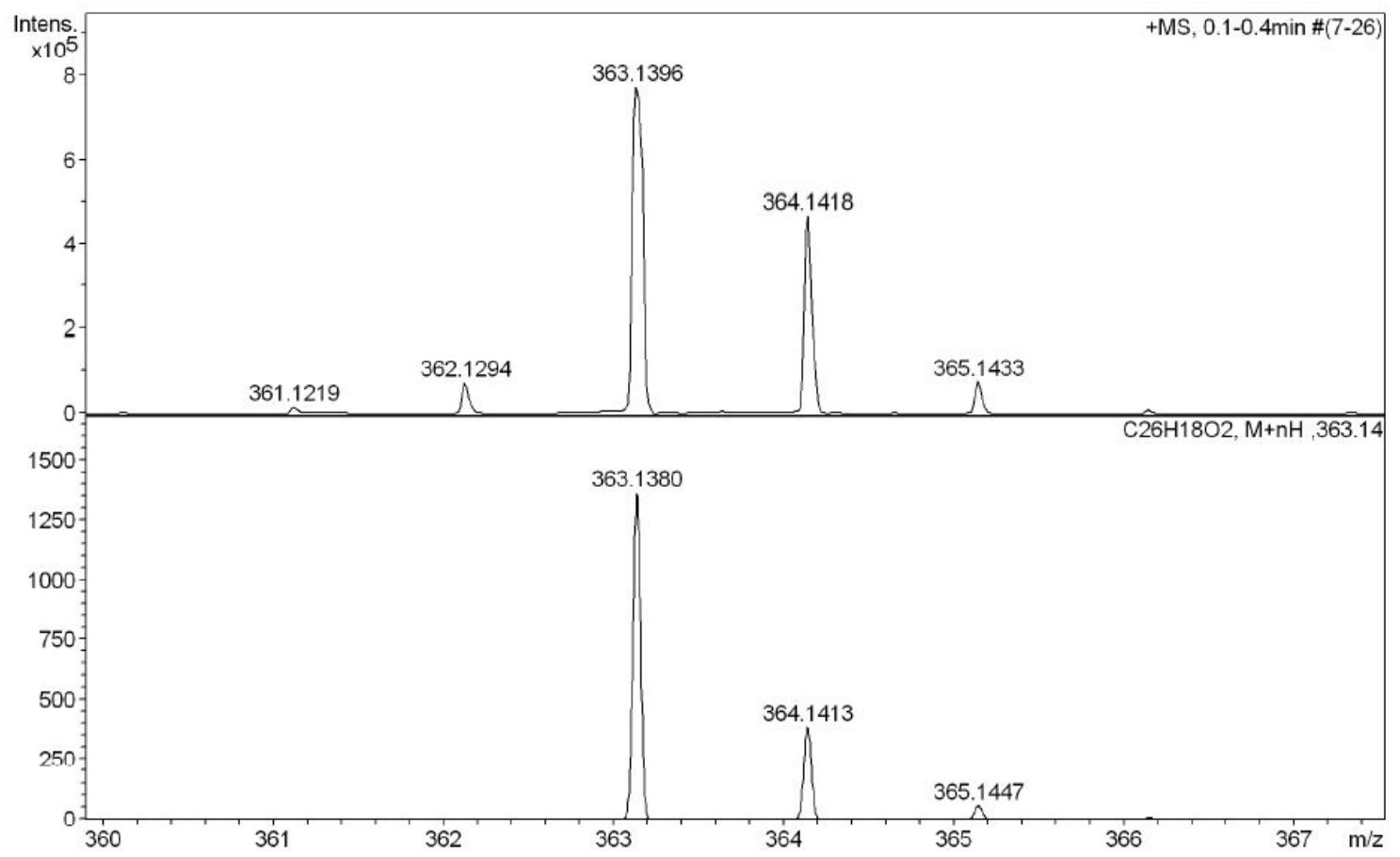

Fig. S6. HRMS spectrum of 9-(3,5-dihydroxyphenyl)triptycene (1) in $\mathrm{CDCl}_{3}$. 


\section{b. 9-(3,5-dimethoxyphenyl)triptycene (3)}

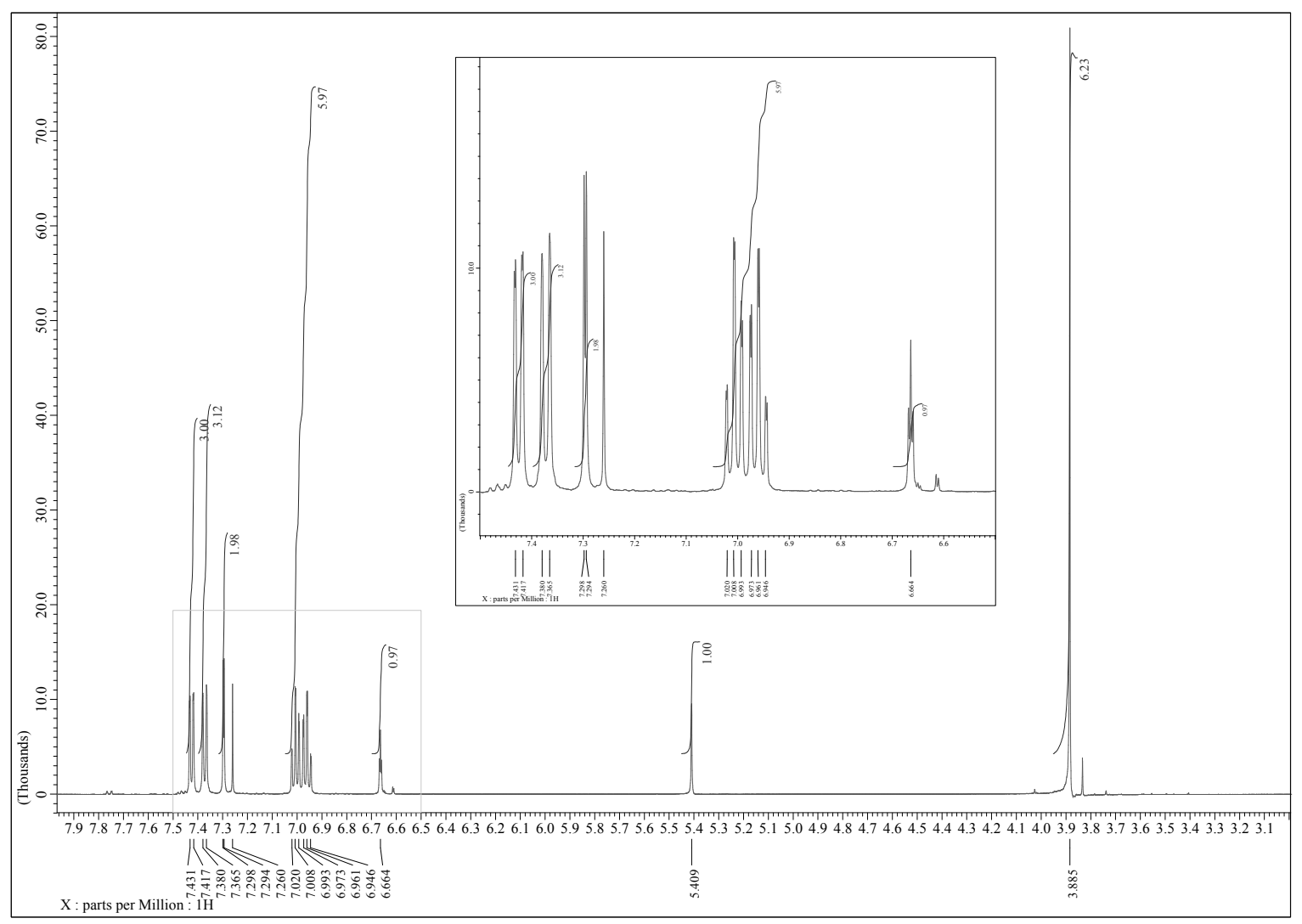

Fig. S7. ${ }^{1} \mathrm{H}$ NMR spectrum of 9-(3,5-dimethoxyphenyl)triptycene (3) in $\mathrm{CDCl}_{3}$.

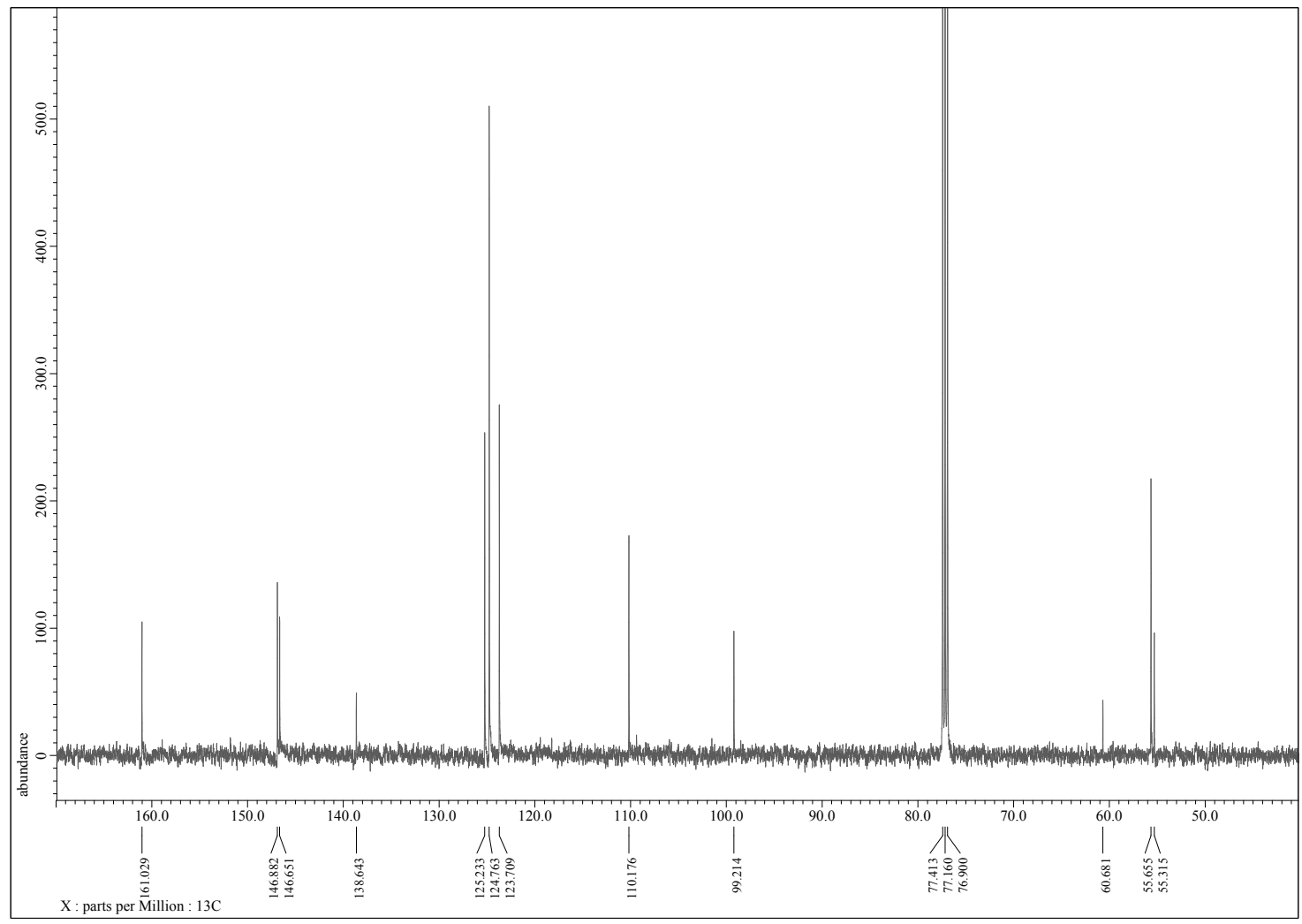

Fig. S8. ${ }^{13} \mathrm{C}$ NMR spectrum of 9-(3,5-dimethoxyphenyl)triptycene (3) in $\mathrm{CDCl}_{3}$. 

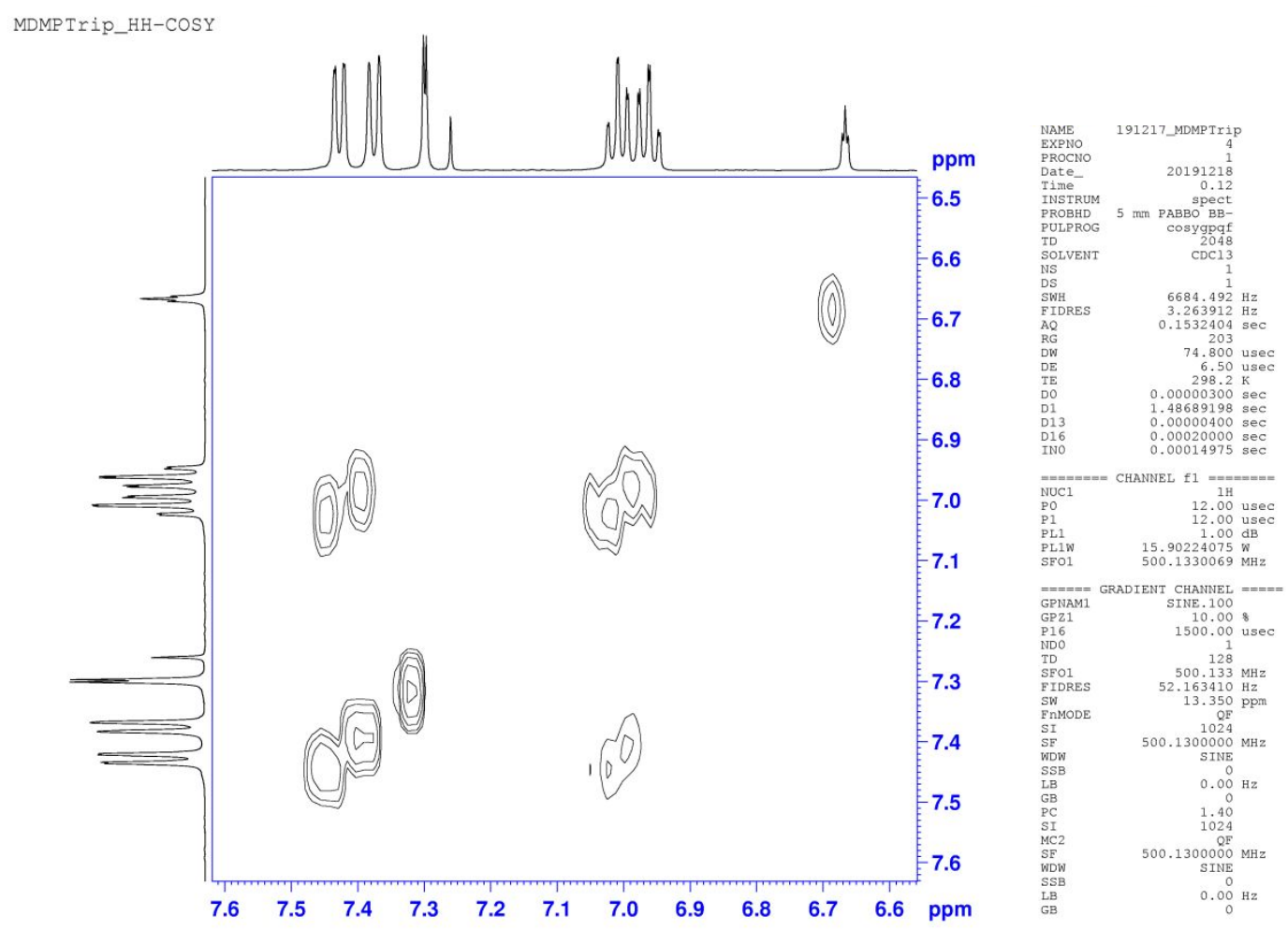

Fig. S9. ${ }^{1} \mathrm{H}-{ }^{1} \mathrm{H}$ COSY NMR spectrum (aromatic region) of 9-(3,5-dimethoxyphenyl)triptycene (3).

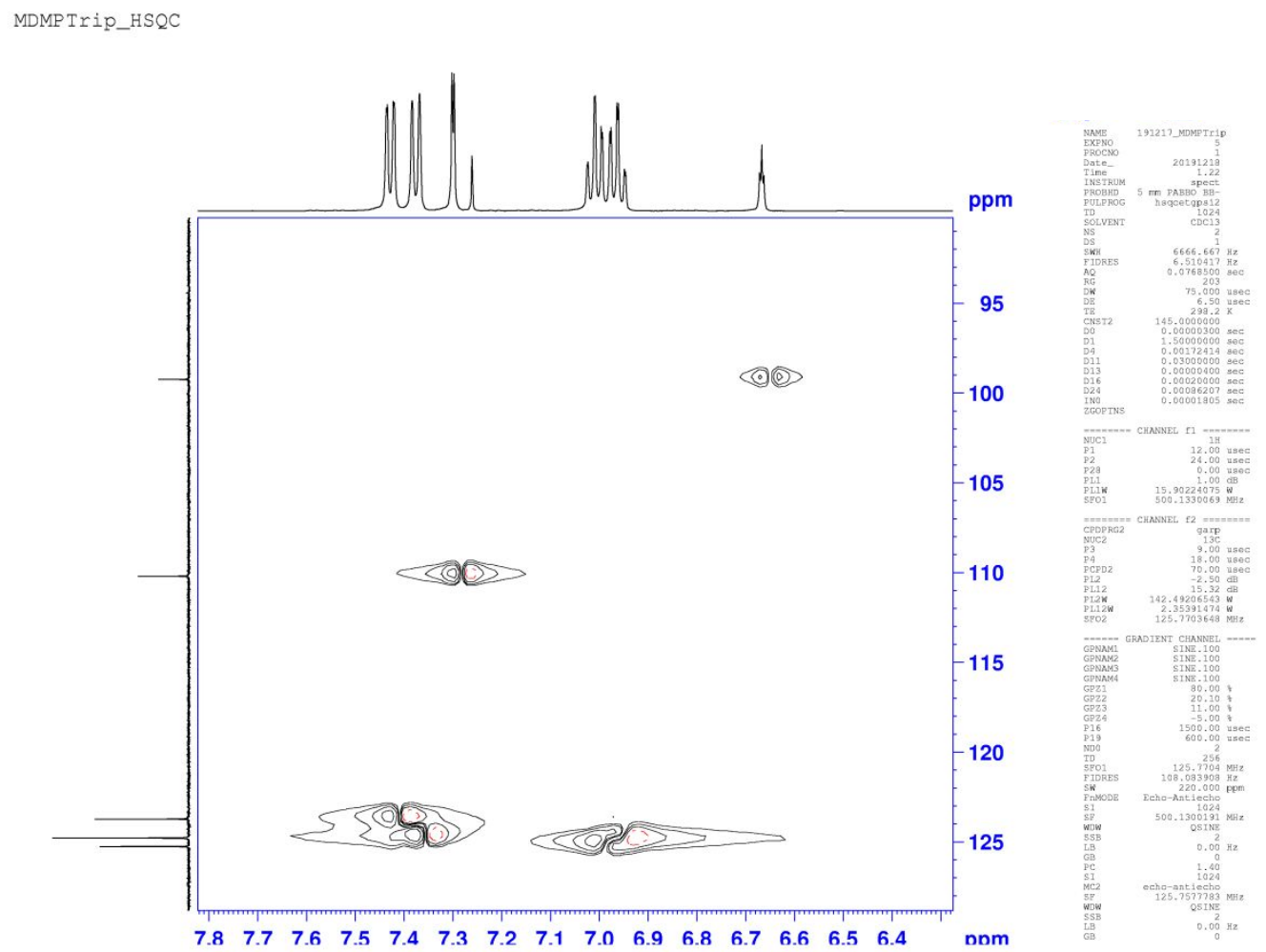

Fig. S10. ${ }^{1} \mathrm{H}-{ }^{13} \mathrm{C}$ HSQC NMR spectrum (aromatic region) of 9-(3,5-dimethoxyphenyl)triptycene (3). 

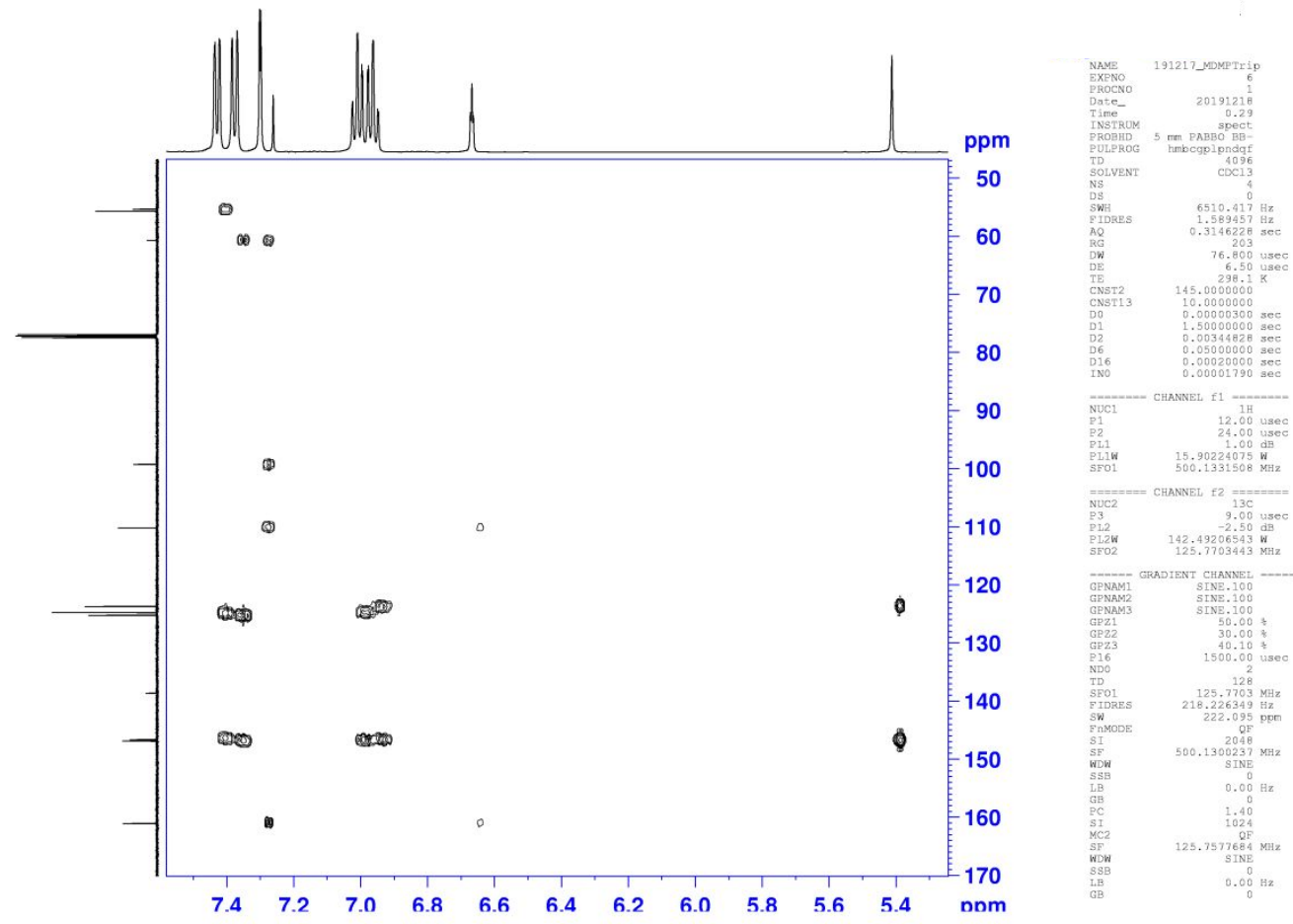

Fig. S11. ${ }^{1} \mathrm{H}-{ }^{13} \mathrm{C}$ HMBC NMR spectrum of 9-(3,5-dimethoxyphenyl)triptycene (3).

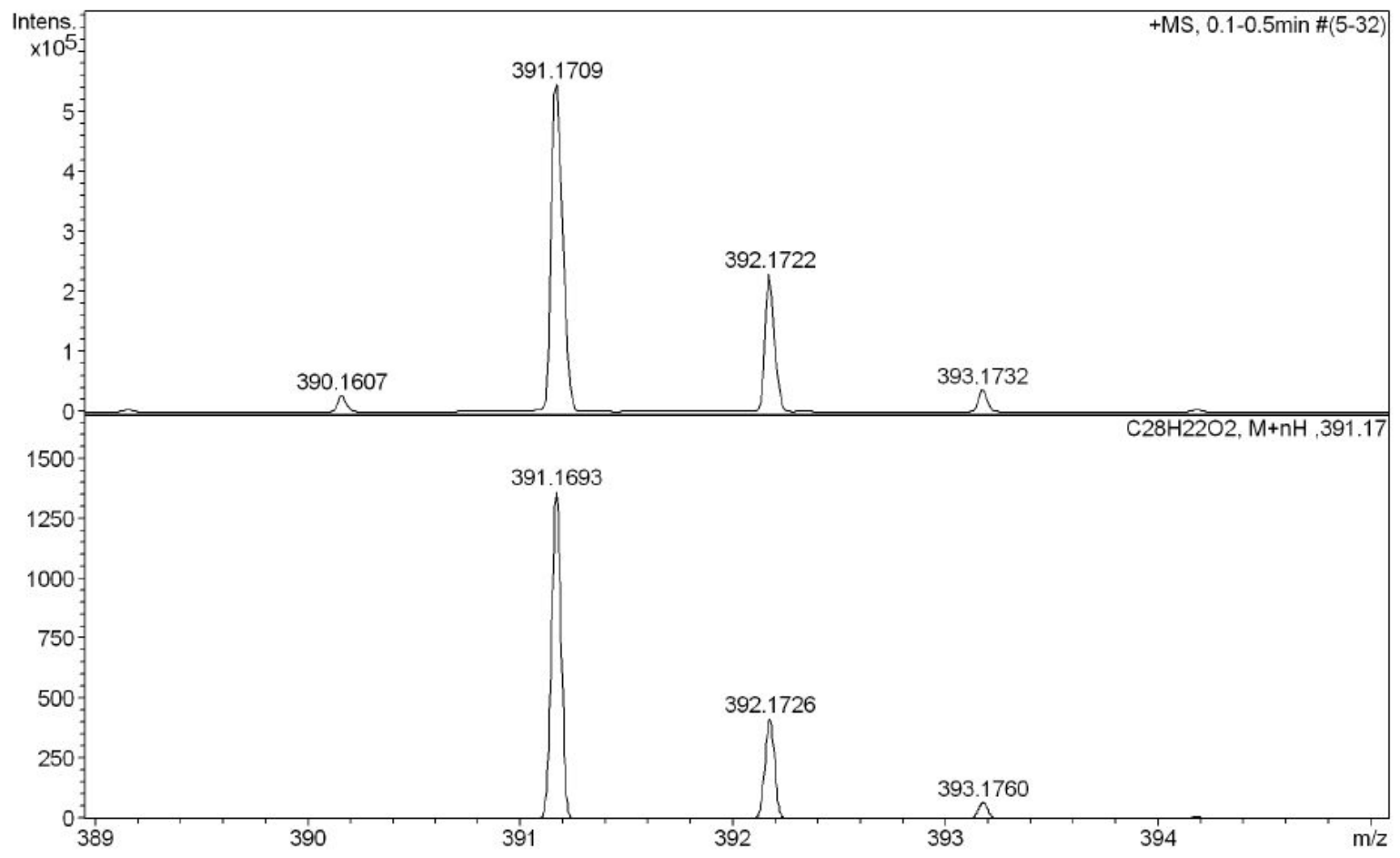

Fig. S12. HRMS spectrum of 9-(3,5-dimethoxyphenyl)triptycene (3) in $\mathrm{CDCl}_{3}$. 


\section{Details of X-ray Crystallography}

\section{Summary}

Table S1. Summary of Crystal Data

\begin{tabular}{|c|c|c|c|c|}
\hline \multicolumn{2}{|l|}{ Crystal } & I (1) & II (1·3MeOH) & III $\left(1 \cdot \mathrm{H}_{2} \mathrm{O}\right)$ \\
\hline \multicolumn{2}{|l|}{ CCDC \# } & 1960888 & 1960889 & 1960890 \\
\hline \multicolumn{2}{|l|}{ Temp / K } & $100(2)$ & $100(2)$ & $100(2)$ \\
\hline \multicolumn{2}{|l|}{ Empirical formula } & $\mathrm{C}_{26} \mathrm{H}_{18} \mathrm{O}_{2}$ & $\mathrm{C}_{29} \mathrm{H}_{30} \mathrm{O}_{5}$ & $\mathrm{C}_{26} \mathrm{H}_{20} \mathrm{O}_{3}$ \\
\hline \multicolumn{2}{|l|}{ Cryst shape } & prism & prism & plate \\
\hline \multicolumn{2}{|l|}{ Cryst color } & colorless & colorless & colorless \\
\hline \multicolumn{2}{|l|}{ Cryst size } & $\begin{array}{c}0.41 \times 0.40 \times 0.37 \\
\mathrm{~mm}^{3}\end{array}$ & $\begin{array}{c}0.37 \times 0.23 \times 0.16 \\
\mathrm{~mm}^{3}\end{array}$ & $\begin{array}{c}0.208 \times 0.196 \times 0.079 \\
\mathrm{~mm}^{3}\end{array}$ \\
\hline \multicolumn{2}{|c|}{ Formula weight / $\mathrm{g} \mathrm{mol}^{-1}$} & 362.40 & 458.53 & 380.42 \\
\hline \multicolumn{2}{|c|}{ Crystal system } & Orthorhombic & Orthorhombic & Monoclinic \\
\hline \multicolumn{2}{|l|}{ Space group } & Pnma & Pnma & $P 2_{1} / n$ \\
\hline \multicolumn{2}{|l|}{$\mathbf{Z}$} & 4 & 4 & 4 \\
\hline \multirow{7}{*}{ Cell parameter } & $a$ & $15.1868(14) \AA$ & $16.041(4) \AA$ & $10.2040(10) \AA$ \\
\hline & $b$ & $13.3822(12) \AA$ & $13.714(2) \AA$ & $15.2420(10) \AA$ \\
\hline & $c$ & $8.7288(11) \AA$ & $11.3708(19) \AA$ & $13.1660(10) \AA$ \\
\hline & $\alpha$ & $90^{\circ}$ & $90^{\circ}$ & $90^{\circ}$ \\
\hline & $\beta$ & $90^{\circ}$ & $90^{\circ}$ & $109.445(2)^{\circ}$ \\
\hline & $\gamma$ & $90^{\circ}$ & $90^{\circ}$ & $90^{\circ}$ \\
\hline & $V$ & $1774.0(3) \AA^{3}$ & $2501.5(8) \AA^{3}$ & $1930.9(3) \AA^{3}$ \\
\hline \multicolumn{2}{|l|}{ Calculated density } & $1.357 \mathrm{Mg} / \mathrm{m}^{3}$ & $1.218 \mathrm{Mg} / \mathrm{m}^{3}$ & $1.309 \mathrm{Mg} / \mathrm{m}^{3}$ \\
\hline \multicolumn{2}{|l|}{$\mathbf{F}(000)$} & 760 & 976 & 800 \\
\hline \multicolumn{2}{|l|}{ Absorption coefficient } & $0.085 \mathrm{~mm}^{-1}$ & $0.082 \mathrm{~mm}^{-1}$ & $0.085 \mathrm{~mm}^{-1}$ \\
\hline \multicolumn{2}{|l|}{$\begin{array}{c}\theta \text { range for collecn } \\
\text { (deg) }\end{array}$} & 2.682 to $25.000^{\circ}$ & 2.195 to $24.995^{\circ}$ & 2.116 to $25.907^{\circ}$ \\
\hline \multicolumn{2}{|l|}{ Index ranges } & $\begin{array}{c}-18<=\mathrm{h}<=18 \\
-15<=\mathrm{k}<=15 \\
-10<=\mathrm{l}<=10\end{array}$ & $\begin{array}{c}-19<=\mathrm{h}<=19 \\
-16<=\mathrm{k}<=16 \\
-13<=\mathrm{k}<=13\end{array}$ & $\begin{array}{c}-12<=\mathrm{h}<=12, \\
-18<=\mathrm{k}<=18 \\
-16<=\mathrm{k}<=16\end{array}$ \\
\hline \multicolumn{2}{|l|}{ Reflections collected } & 13718 & 20196 & 17830 \\
\hline \multicolumn{2}{|l|}{$\begin{array}{l}\text { Independent } \\
\text { reflections }\end{array}$} & $\begin{array}{c}1631[\mathrm{R}(\mathrm{int})= \\
0.0564]\end{array}$ & $\begin{array}{c}2306[\mathrm{R}(\text { int })= \\
0.1302]\end{array}$ & $\begin{array}{c}3636[\mathrm{R}(\mathrm{int})= \\
0.0238]\end{array}$ \\
\hline \multicolumn{2}{|l|}{ Completeness } & $99.8 \%$ & $100.0 \%$ & $98.7 \%$ \\
\hline \multicolumn{2}{|l|}{ Goodness-of-fit on $F^{2}$} & 1.263 & 1.107 & 1.030 \\
\hline \multicolumn{2}{|l|}{$\begin{array}{c}\text { Final R indices } \\
{[\mathrm{I}>2 \operatorname{sigma}(\mathrm{I})]}\end{array}$} & $\begin{array}{c}\mathrm{R} 1=0.0928, \mathrm{wR} 2= \\
0.2223\end{array}$ & $\begin{array}{c}\mathrm{R} 1=0.0937, \mathrm{wR} 2= \\
0.2377\end{array}$ & $\begin{array}{c}\mathrm{R} 1=0.0346, \mathrm{wR} 2= \\
0.0866\end{array}$ \\
\hline \multicolumn{2}{|l|}{$\mathbf{R}$ indices (all data) } & $\begin{array}{c}\mathrm{R} 1=0.1125, \mathrm{wR} 2= \\
0.2350\end{array}$ & $\begin{array}{c}\mathrm{R} 1=0.1221, \mathrm{wR} 2= \\
0.2589\end{array}$ & $\begin{array}{c}\mathrm{R} 1=0.0384, \mathrm{wR} 2= \\
0.0894\end{array}$ \\
\hline \multicolumn{2}{|l|}{$\begin{array}{l}\text { Largest diff. peak and } \\
\text { hole }\end{array}$} & $\begin{array}{c}0.445 \text { and }-0.553 \\
\text { e. } \AA^{-3}\end{array}$ & $\begin{array}{c}0.589 \text { and }-0.375 \\
\text { e. } \AA^{-3}\end{array}$ & $\begin{array}{c}0.244 \text { and }-0.317 \\
\text { e. } \AA^{-3}\end{array}$ \\
\hline
\end{tabular}




\section{ORTEP Drawing}

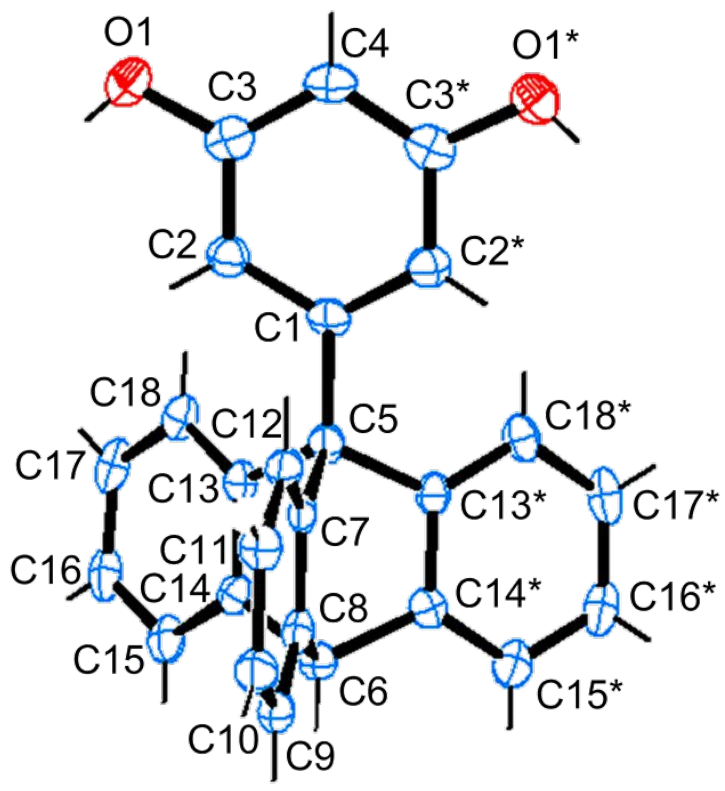

Fig. S13. ORTEP drawing of 9-(3,5-dihydroxyphenyl)triptycene (1) anhydrate (Crystal I) (50\% probability thermal ellipsoids)
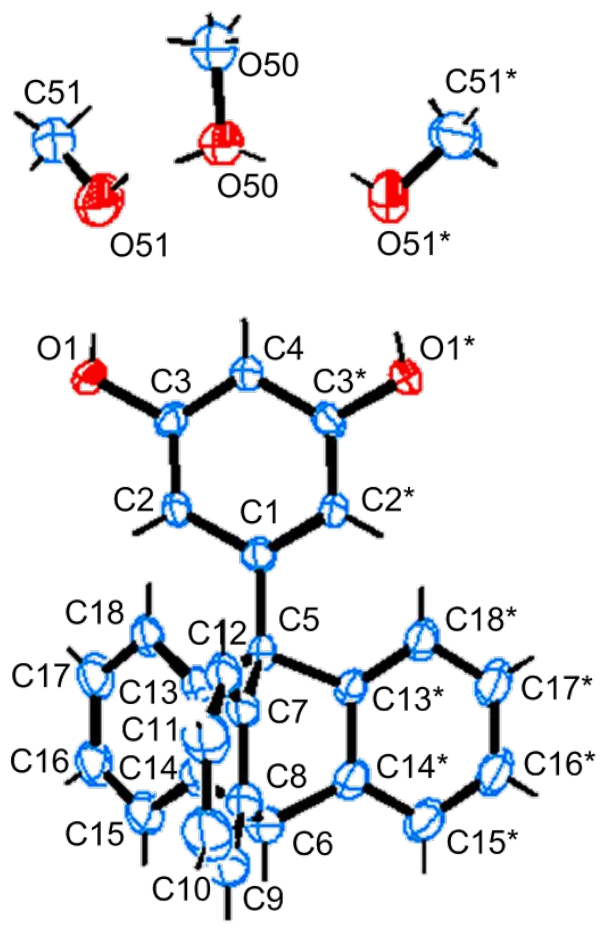

Fig. S14. ORTEP drawing of 9-(3,5-dihydroxyphenyl)triptycene (1) with tree methanols (Crystal II) (50\% probability thermal ellipsoids) 

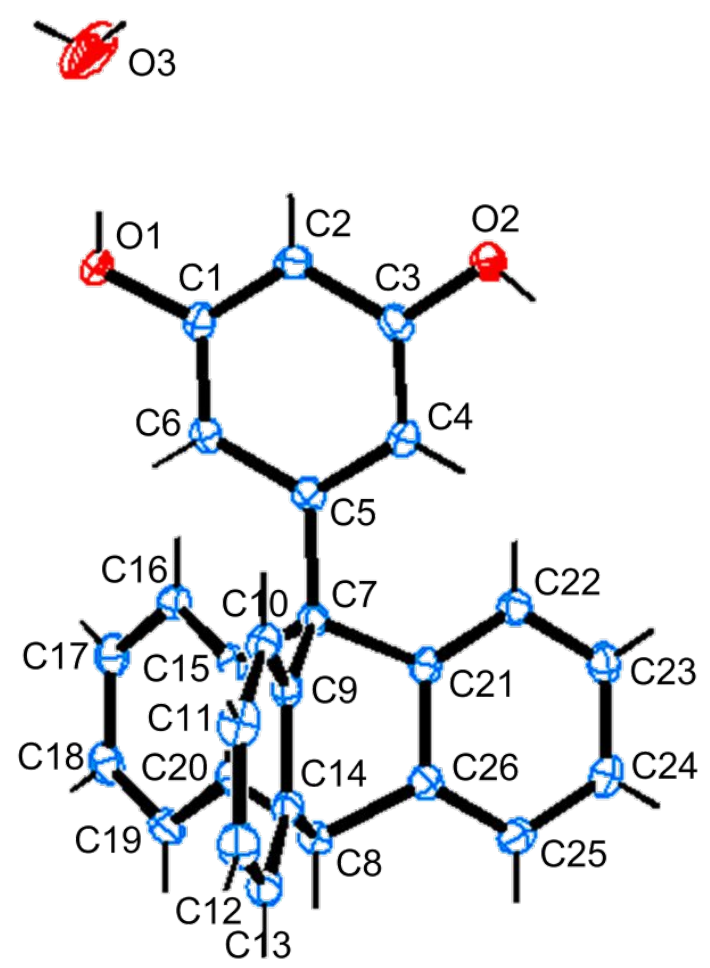

Fig. S15. ORTEP drawing of 9-(3,5-dihydroxyphenyl)triptycene (1) hydrate (Crystal III) (50\% probability thermal ellipsoids) 


\section{Temperature dependent ${ }^{1}$ H NMR Study}

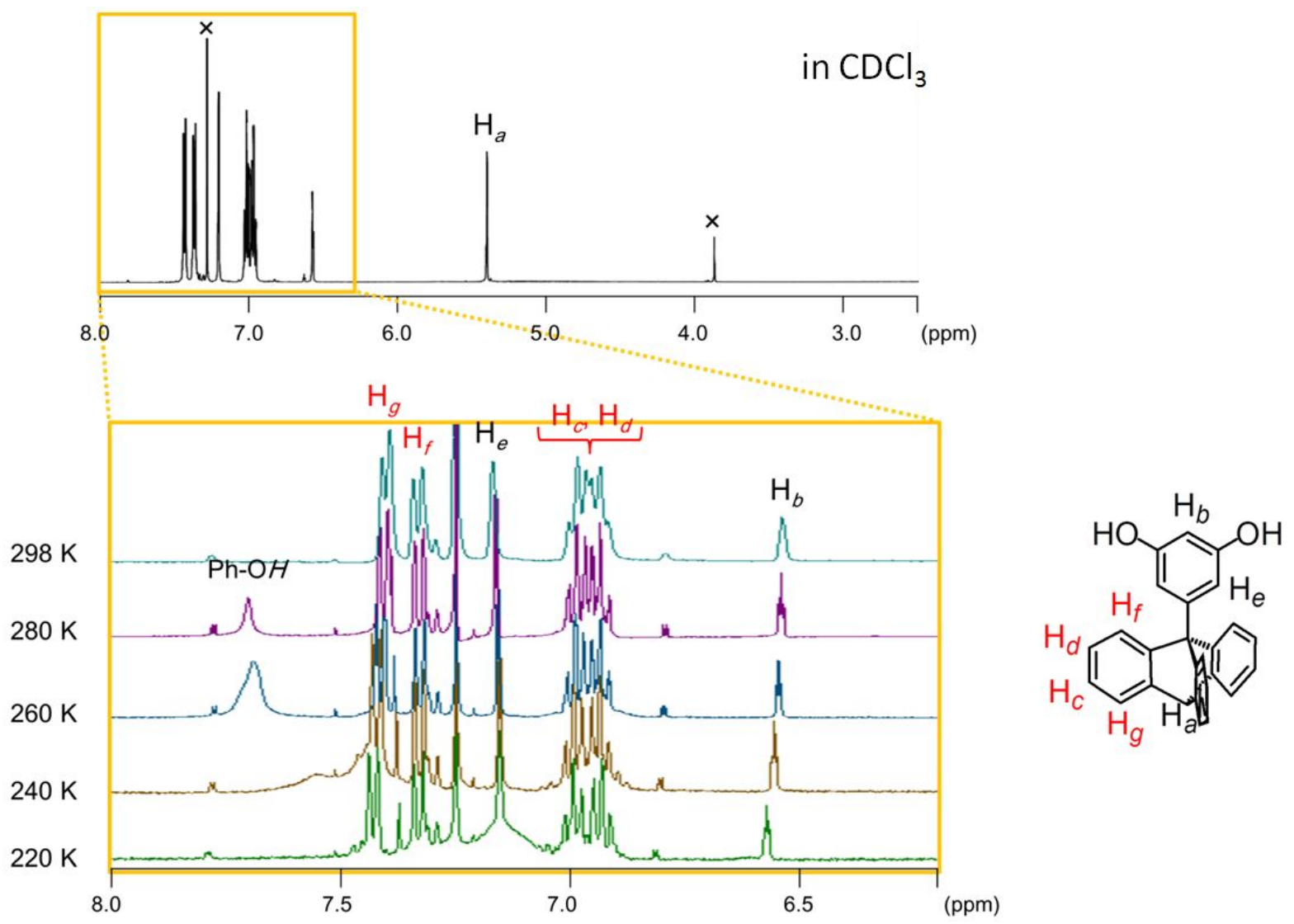

Figure S16. Temperature dependent ${ }^{1} \mathrm{H}$ NMR spectra of $\mathbf{1}$. Signal assignments were also indicated. 


\section{Solid-state ${ }^{13} \mathrm{C}$ CP/MAS NMR Study}

The present study aims to realize a hitherto unknown crystalline multiple molecular gear system that can exhibit correlated rotation of triptycyl groups in a crystalline state. $\mathbf{1} \cdot \mathrm{H}_{2} \mathrm{O}$ (crystal III) is a candidate structure that could be used to achieve such a system.

The dynamics of the triptycyl groups in the crystals were preliminary investigated by solid-state ${ }^{13} \mathrm{C}$ NMR studies of powdered samples of $\mathbf{1} \cdot \mathrm{H}_{2} \mathrm{O}$ (crystal III) as well as $\mathbf{1}$ (crystal I), which was used as a reference. The high purity of the powdered samples were confirmed by PXRD studies. Figure S17a shows the ${ }^{13} \mathrm{C}$ cross-polarization magic angle spinning (CP/MAS) NMR spectra of powdered $\mathbf{1}$ (crystal I) with 6 $\mathrm{kHz}$ spinning of the sample at $296 \mathrm{~K}$. The observed signals due to the triptycyl $\mathrm{C}-\mathrm{H}$, marked with the symbol A in Figure S17a, can be attributed to somewhat rotationally hindered groups, because many signals are distinct and exhibit anisotropy that is detected by the presence of spinning sidebands, indicated by asterisks in Figure S17. Figure S17b shows ${ }^{13} \mathrm{C} \mathrm{CP} /$ MAS NMR spectra of powdered $\mathbf{1} \cdot \mathrm{H}_{2} \mathrm{O}$ (crystal III) at $6 \mathrm{kHz}$ and at $296 \mathrm{~K}$. Although the signals attributed to the triptycyl carbons (marked with symbol A) are more overlapped than the corresponding signals observed in crystal I due to similar environments of CHs in the triptycyls inside the crystal on the same line of reasoning as for $\mathbf{1}$ (crystal I). We note that slow motion of the dynamics of the triptycyl could not be observed by this method. Temperature dependent relaxation time of ${ }^{13} \mathrm{C}$ nuclei and/or ${ }^{2} \mathrm{H}$ NMR study of deuterated triptycene are required to estimate the dynamics more precisely.

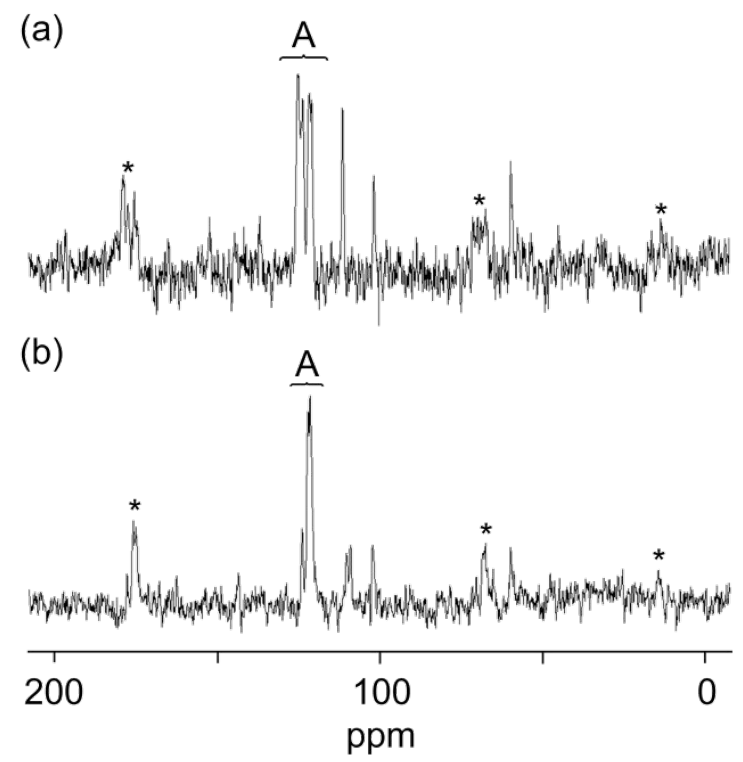

Figure S17. Solid-state ${ }^{13} \mathrm{C} \mathrm{CP} / \mathrm{MAS}$ (MAS rate: $6 \mathrm{kHz}$ ) spectra of the powder sample at $296 \mathrm{~K}$ : (a) 1 (crystal I) (b) $\mathbf{1} \cdot \mathrm{H}_{2} \mathrm{O}$ (crystal III). 


\section{Temperature dependent PXRD Study}

(a)

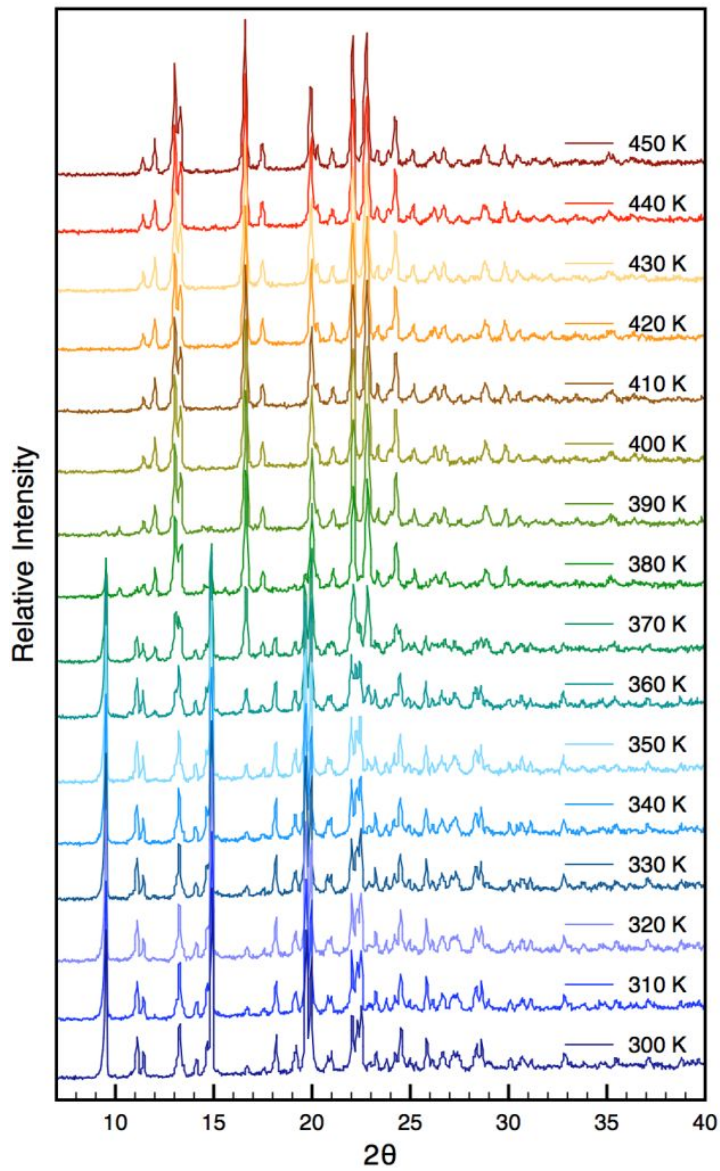

(b)

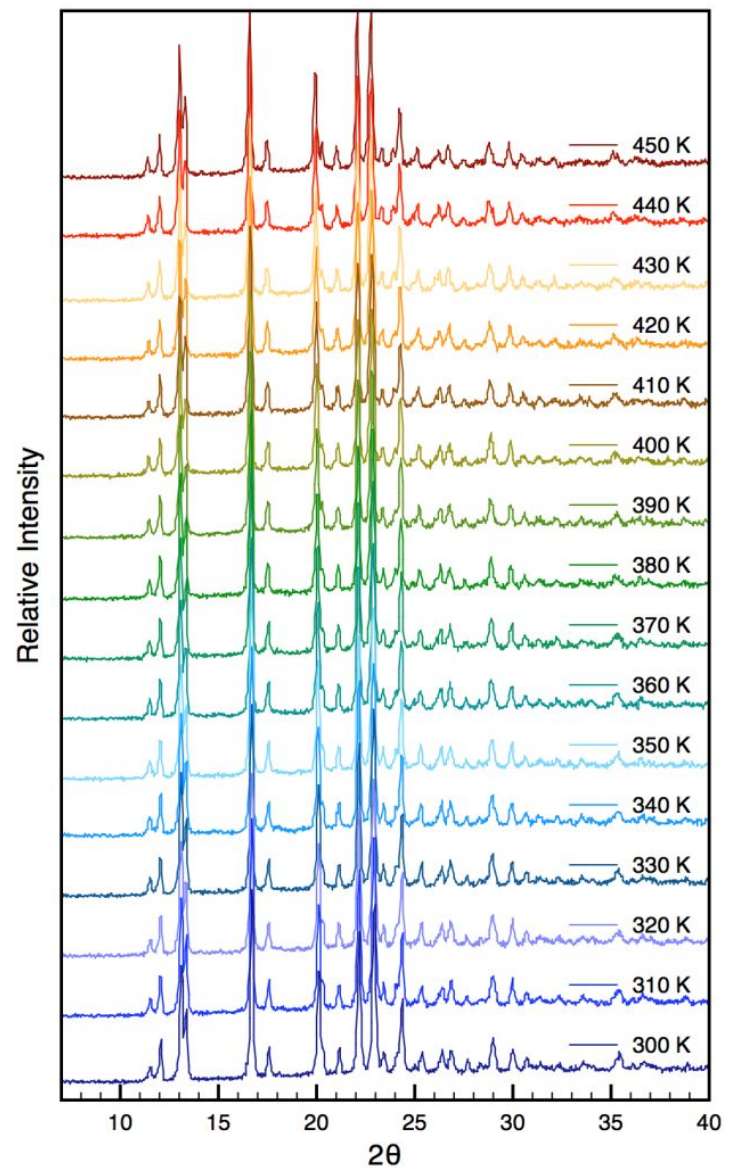

Figure S18. Temperature dependent PXRD patterns of $\mathbf{1} \cdot \mathrm{H}_{2} \mathrm{O}$ (crystal III) (rate: $20 \mathrm{~K} /$ minutes): (a) heating process (from $300 \mathrm{~K}$ to $450 \mathrm{~K}$ ); (b) cooling process (from $450 \mathrm{~K}$ to $300 \mathrm{~K}$ ). 